\title{
THE DESIGN OF INTELLIGENT CHARGER OF ELECTRIC VEHICLE UNDER THE CONTROL OF MICROCONTROLLERS
}

\author{
Hongyan Liu \\ Shandong Polytechnic, Jinan 250104, China. \\ Email: liuhongyanjndx@163.com
}

\begin{abstract}
To explore the mechanism and effect of the intelligent charger of electric vehicle under the control of microcontroller (MCU), the working effect of the intelligent charger of electric vehicle was discussed by constructing the charger model of electric vehicle based on STC12C5204AD MCU and the simulation experiment method. The specific energy density of the battery for the charger under the action of STC12C5204AD, 8xC752, and C504 MCU and the charging times for the battery life under different single chip computers were mainly studied. Moreover, the effect and mechanism of the intelligent charger and the common charger were explored. The research results showed that the battery based on STC12C5204AD MCU had little effect on the change of specific energy of battery, the specific energy basically maintained the initial value, and the effect was prominent. Compared with 8xC752 and C504 MCU, STC12C5204AD MCU had the most prominent effect on delaying the decline of battery life under the same charging times. In contrast with the general charger, the intelligent charger would change its charging voltage according to the change of the rated voltage of the battery to maintain the maximum protection of the battery life. The ordinary battery could maintain a certain value without random change. To sum up, compared with the common electric vehicle charger, the intelligent charger has obvious advantages, which significantly improves the protection of the battery and delays the decline of it. The intelligent electric vehicle charger based on STC12C5204AD MCU has outstanding effect. The model of the intelligent electric vehicle charger based on STC12C5204AD meets the high standard requirements of the electric vehicle for the charger. The research on the intelligent charger of electric vehicle under the control of MCU has a positive effect on the follow-up research.
\end{abstract}

Keywords: MCU; Charger; Electric Vehicle.

\section{Introduction}

With the development of society, the pollution is more and more serious, and the global air is greatly polluted. Therefore, improving the environment has become a global unified theme. The use of electric vehicles greatly reduces the air environment pollution caused by automobiles. Electric vehicles use electricity as the energy for their work, which is clean and free of exhaust emissions [1]. Due to the global warming temperature rising, the research on energy saving and environmental protection is very popular and open, which has become the current mainstream research direction [2]. The research on electric vehicles includes all levels, including the most important part of charger [3]. The energy flow is generally achieved through conversion. The most important and clean energy source is electric energy. For China, although the electric resources are relatively scarce, the environmental pollution is very serious [4]. Therefore, it is extremely important to use the electric resources efficiently for achieving the stable, safe, and long-term operation of electric vehicles. Most electric vehicles realize the electric energy conversion through energy charging [5].

Electric energy is widely used in daily life. No matter in military, aerospace, industry, and daily life, it can become the power to make many things after conversion and processing. At present, electric energy has become the most important energy demand of the society [6], but there are still many problems in the research and development of electric vehicle chargers, such as low efficiency and safety.

The key is that it will cause great damage to the battery and reduce the service life of the battery of the electric vehicle [7]. If there is no efficient and intelligent charger technology, it is impossible to store the electric energy according to the actual situation and not damage the battery [8-10]. Because only the intelligent charger can protect the battery to the greatest extent and delay the decline of the battery to meet the needs of intelligent charging of electric vehicles. The working effect of the intelligent charger for electric vehicle is studied by building the charger model of electric vehicle based on 
microcontroller (MCU) and the simulation experiment method. The key factors such as battery life and specific energy of the charger are mainly studied, so that the function and value of the charger system can be qualitatively and quantitatively analyzed.

The essence of charging is the transmission of energy. Efficient and intelligent transmission technology is extremely important for any field research. Hence, the research on an intelligent charger system is indispensable for the use and popularization of electric vehicles [11]. Electric energy is a very huge clean energy with high relative value ratio. The most important thing at present is to find efficient and intelligent electric vehicle charger technology, while it is still in a relatively primary stage. Consequently, the research focus of this paper is to build and design a system to meet the intelligent charging needs of electric vehicles.

In conclusion, the working effect of the intelligent charger of electric vehicle is discussed by building the charger model of electric vehicle based on MCU and the simulation experiment method. The research shows that the intelligent charger for electric vehicle based on STC12C5204AD is more prominent than the ordinary charger and other intelligent chargers for battery protection. The MCU model constructed meets the requirements for electric vehicle charging and battery protection. The innovation of this paper lies in that it analyzes the core parts of charger in electric vehicle charging.

\section{Method \\ 2.1 Design requirements of intelligent
charger for electric vehicles}

Rapidity: the most important parameter for the battery charger of electric vehicle is the time required for charging, which is extremely core for the charger [12]. When the charging time is less than 3 hours, it belongs to the fast charging technology. For a general electric vehicle, the size of the charging current used by the battery is often closely related to the capacity of the battery itself. The result of battery capacity in 10 hours is usually taken as the current setting requirement for charging [13].

Out gassing amount and out gassing rate: for the charging process of electric vehicle, out gassing amount and out gassing rate are the core design parameters, which have a great impact on the life of battery [14]. Out gassing amount generally refers to the total amount of gas produced by anode and cathode in the complete charging process of electric vehicle battery under standard atmospheric pressure, with the unit of liter and out gassing. The out gassing rate refers to the total amount of gas produced by anode and cathode in a certain charging link under a standard atmospheric pressure [15], and the unit is liter/minute. In order to improve the protection for battery and extend the working time of battery, reducing the out gassing amount is a very important work. Figure 1 shows the charging voltage and current curve of battery.

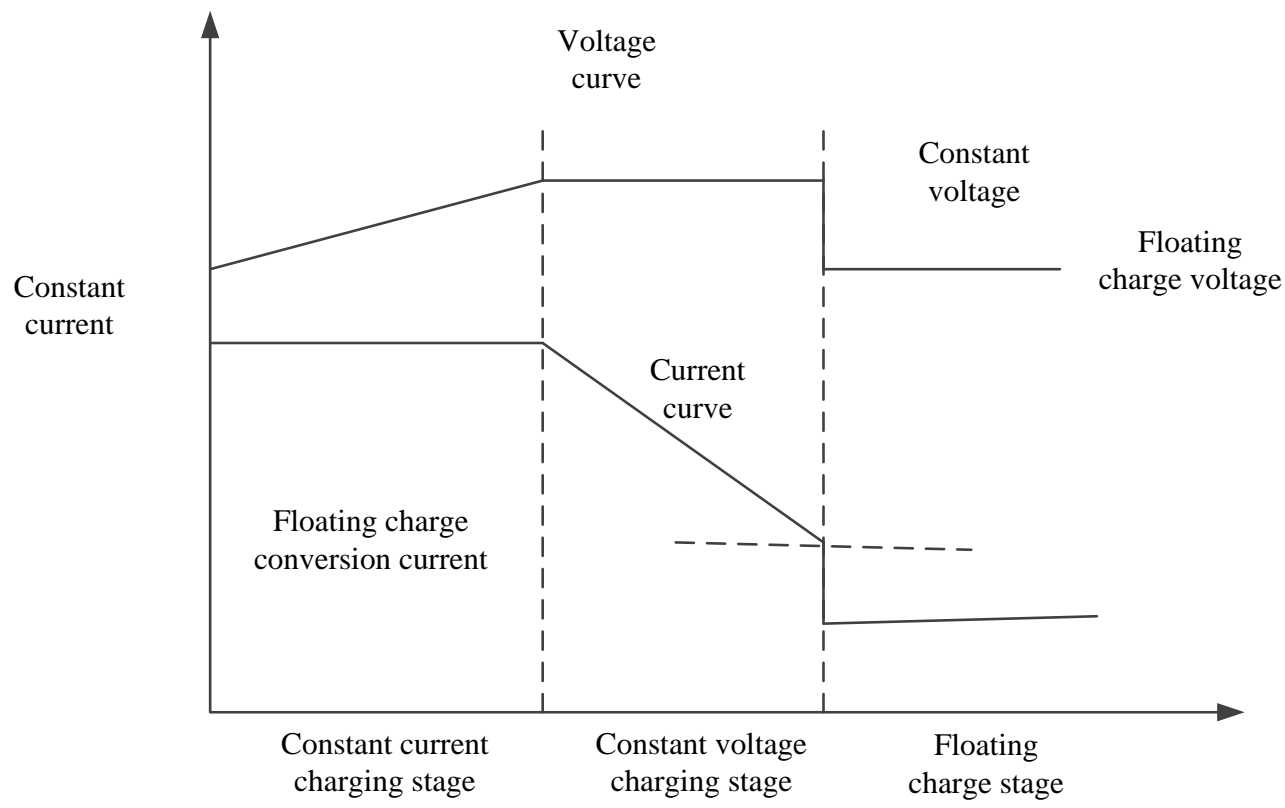

Figure 1: Charging voltage and current of battery

Temperature: in general, the electric vehicle will have a relatively obvious temperature rise in the process of charging. The rise of temperature will lead to the imagination of water loss in the battery, which will lead to the increase of electrolyte concentration and polarization. At last, it will greatly affect the life of the battery, and accelerate the decline of the battery. The battery charger technology is required to have very strict standards and settings for the temperature control. The change of temperature shall be controlled within a certain range to ensure no obvious impact on the battery. 
According to the research of the actual situation, the temperature of the electrolyte must not exceed $38^{\circ} \mathrm{C}$ [16].

Life: for the electric vehicle itself and the battery and charging technology, the life of the battery is the core requirement of the research. During the charging process of the electric vehicle, there will be a relatively obvious temperature rise [17]. The rise of the temperature will cause a great impact on the life of the battery, as well as the number of cycles charging of the battery since the battery itself is charged with a certain time.

\subsection{Design principle of intelligent charger}

Figure 2 shows the intelligent charging principle of electric vehicle. According to the accurate inspection and analysis of the key information of lead-acid battery, the charger system of electric vehicle transmits the searched information to the MCU system after processing and analysis through the converter.

The function of MCU is to analyze and study the battery state of electric vehicle after receiving the information, thereby judging and collecting the charging mode. The goal is to adjust and control the charging voltage and current of the charger, and monitor and detect the state of the battery in real time in the whole charging process.

More importantly, it can charge the battery of the electric vehicle in the way of pulse charging through the control and adjustment of the MCU system, adjust the battery as much as possible by fitting itself, make battery charging according to the optimal route, and increases the efficiency of the battery.

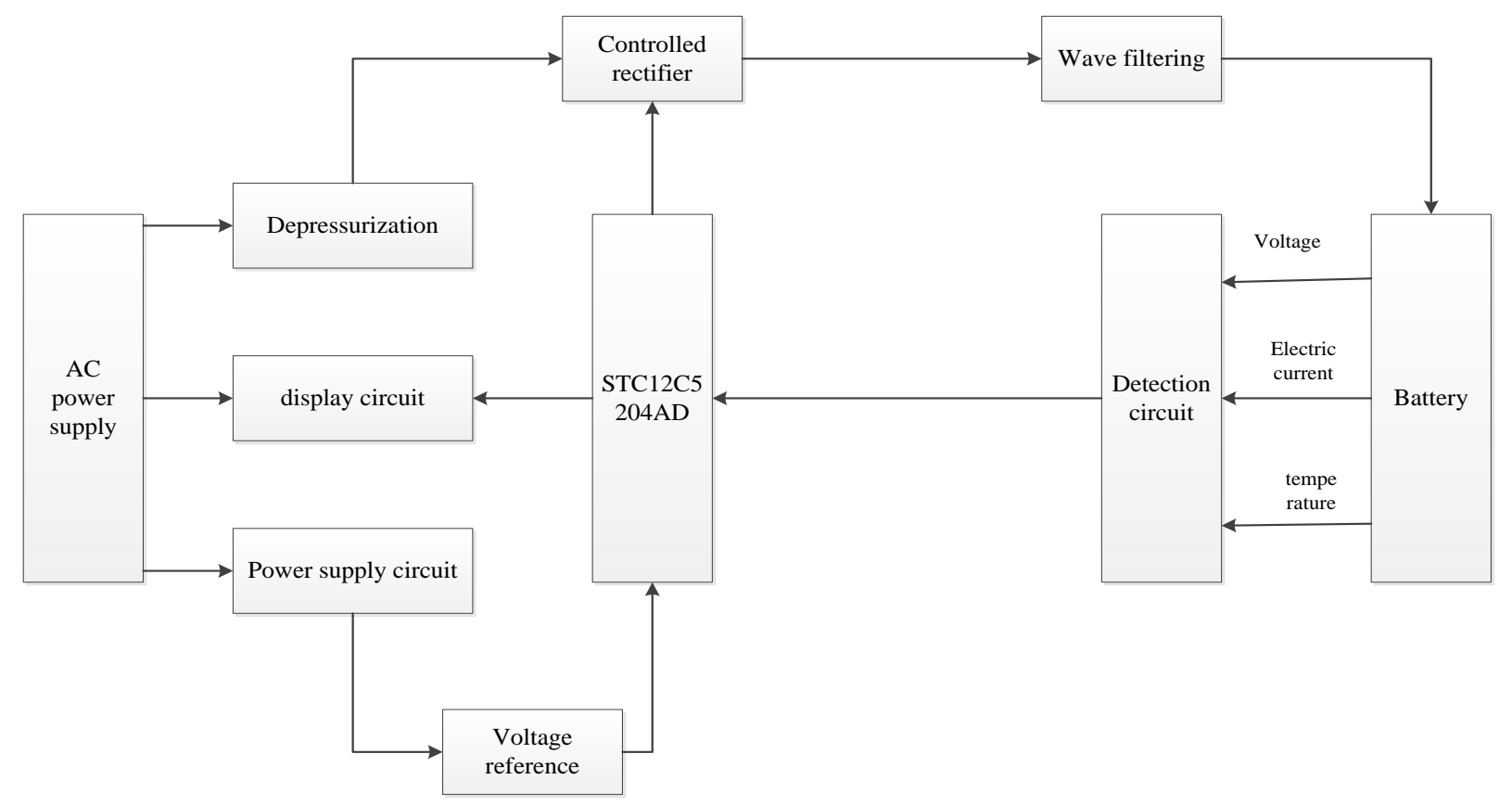

Figure 2: Electric vehicle intelligent charging

Lead acid battery is a kind of experimental equipment which transforms chemical energy into electric energy, which mainly relies on the chemical substances. The reaction of battery is reversible. Both forward reaction and reverse reaction can occur.

Chemical energy can be converted into electric energy, and electric energy can also be converted into chemical energy, corresponding to the change of energy properties. Its core reaction process is as follows:

$$
\mathrm{PbSO}_{4}+2 \mathrm{H}_{2} \mathrm{O} \rightarrow \mathrm{PbO}_{2}+\mathrm{H}_{2} \mathrm{SO}_{4}+\mathrm{Pb}
$$

\section{$2.3 \mathrm{MCU}$}

In the intelligent charger system of electric vehicle, MCU needs to be able to meet the requirements of power supply, crystal vibration, and grounding. Only by meeting these requirements can the stable working state of the MCU system be ensured. The principle frame of the current controlled switching power supply is shown in Figure 3. The MCU system is the core control link of the electric vehicle charger. STC12C5204AD MCU is used as the control core of the charger in this paper. For the function and state of MCU, further understanding can help maximizing the control role of MCU. 


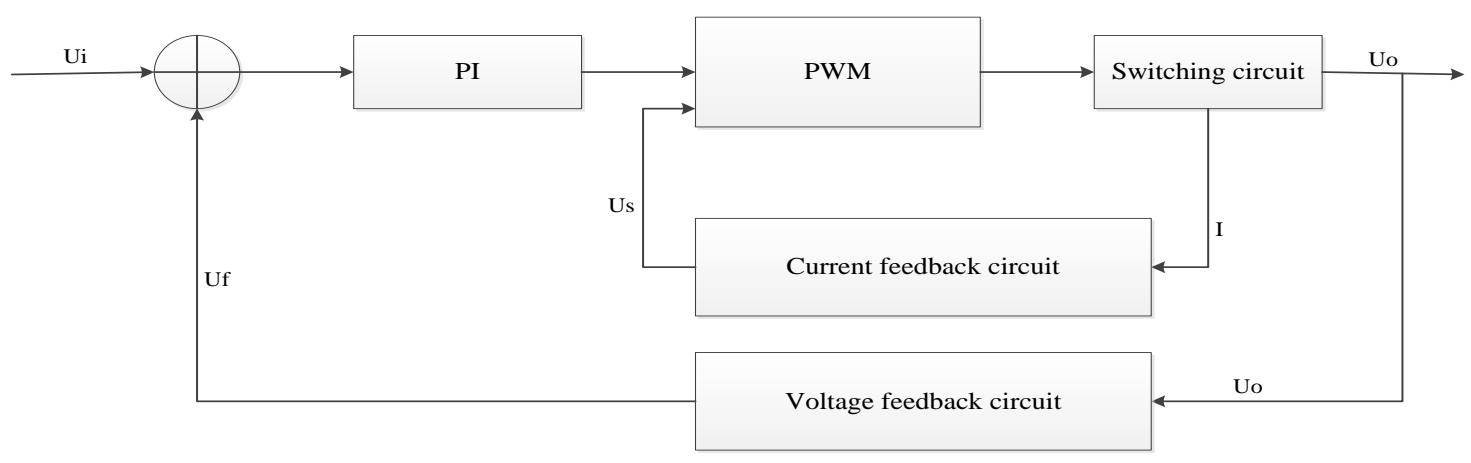

Figure 3: Principle frame of current controlled switching power supply

The main working process and content of MCU is to collect and process the data of electric power and voltage at every moment, and then transmit it to the MCU system through the analysis of converter, so as to judge and adopt the charging mode, and adjust and control the charging voltage and current of charger. It also aims at balancing the capacity state of battery in the whole charging process and prevent battery damage due to improper charging. In addition, according to different batteries, MCU can adjust itself for fitting as much as possible and charge the battery according to the optimal route, thereby increasing the efficiency of the battery and meet the charging demand and safety protection of the non-charging battery. If the battery is unbalanced, MCU will adjust it according to the actual situation, charge less if there is more electricity, and charge more if there is less electricity.

\subsection{Determination of main parameters of intelligent charger}

In the use of lead-acid batteries for electric vehicles, due to the high frequency of battery cycle charge and discharge, to ensure the service life of the battery, it is obviously not enough to rely solely on the technical progress of the battery itself. Practice proves that choosing a reasonable charging mode and designing reliable charging parameters can not only reduce the damage to the battery, but also have certain impact on the battery repair effect.

Maximum charging voltage and minimum charging voltage: the maximum charging voltage is the maximum output voltage of the charger, which is set according to the maximum withstand voltage of the single battery. For sealed lead-acid batteries, the maximum charging voltage that a single cell battery can bear at full charge is not less than $2.35 \mathrm{~V}$ at least, not more than $2.45 \mathrm{~V}$ at most, and the average voltage is about $2.4 \mathrm{~V}$.

Maximum charging current: the setting of the maximum charging current shall be determined according to the actual situation of the battery, and the capacity of the battery is the main basis for reference. Practice verifies that the maximum charging current is generally set to $0.15-0.25$ times of the actual capacity of the battery, which will cause the lowest damage to the battery.

The highest charging current should not be too high, which is a basic principle in the design of fast intelligent charger.

The calculation equation of the best charging current I acceptable to the battery is shown in Eq.2:

$$
\mathrm{I}=\mathrm{I}_{0} \mathrm{e}^{\alpha \mathrm{t}}
$$

I is the maximum charging current at the beginning of charging and $\alpha$ indicates the charge acceptance rate of the battery, which is constant.

If during the charging process, the charge acceptance rate of the battery follows the max curve, then by the end of charging, the charge $\mathrm{C}$ of the battery is shown in Eq.3:

$$
\mathrm{C}=\int_{0}^{\mathrm{t}} \mathrm{Idt}
$$

For a certain discharged battery, i.e. a certain amount of battery to be recovered, the larger the initial maximum charging current I0 is, the higher the charging acceptance rate of the battery will be, the shorter the charging time will be, and the higher the charging efficiency will be.

The design of pulse parameters: in the charging process of the intelligent fast charger, choosing appropriate pulse parameters can effectively remove the polarization phenomenon of the battery.

If the pulse parameters are not properly selected, it will not only fail to depolarize, but also damage the battery to a certain extent.

Therefore, in the charging system, reasonable pulse parameters can be set according to different charging requirements.

Temperature setting: previous practice shows that when charging below $-20^{\circ} \mathrm{C}$, the chemical reaction of active substances inside the battery is slow, and the organic expander adsorbed on the active substances $\mathrm{Pb}$ and $\mathrm{PbSO}_{4}$ will also hinder the dissolution of $\mathrm{PbSO}_{4}$ crystal, so as to reduce the charge receiving capacity.

However, if the temperature of the battery is too high, overcharge and polarization will occur more easily in the charging process. 
With the current technical level, the temperature shall be controlled between $-15^{\circ} \mathrm{C}$ to $-50^{\circ} \mathrm{C}$ as far as possible, and the temperature shall not exceed $40^{\circ} \mathrm{C}$.

\subsection{Charging process analysis}

Design an intelligent electric vehicle charger that meets the requirements. This charger needs to meet the three-stage charging and constant current and constant voltage charging methods. There are different requirements in the process of constant voltage and constant current charging. For the constant voltage charging process, it needs to keep the voltage unchanged, the current will gradually reduce, and it needs to keep the current stable during the constant current charging process. The voltage will gradually increase, and in the process of constant voltage charging, the current will gradually decrease. When the current value decreases below the set value, the charging process will go into the floating charging process.

The first step in the charging process is to change the charging voltage by using charger, change the voltage to the set value by using circuit, and then charge the battery. In the charging process, it is necessary to set the voltage and current value of MCU system in advance, and then switch and disconnect the node time according to the charging process.

For the constant current charging link, it is necessary to keep the current stable during the constant current charging process. As time goes on, the voltage of the battery will rapidly increase. In the constant voltage charging stage, it is necessary to keep the voltage unchanged, and the electric quantity of the battery will gradually increase. When the electric quantity of the battery increases, the current will decrease until the battery is full.

\subsection{Hardware structure of the system}

Figure 4 shows the hardware structure frame of the intelligent charger. The hardware structure of the system consists of step-down circuit, three-stage charging control circuit, MCU minimum system circuit, digital tube circuit, and power supply circuit.

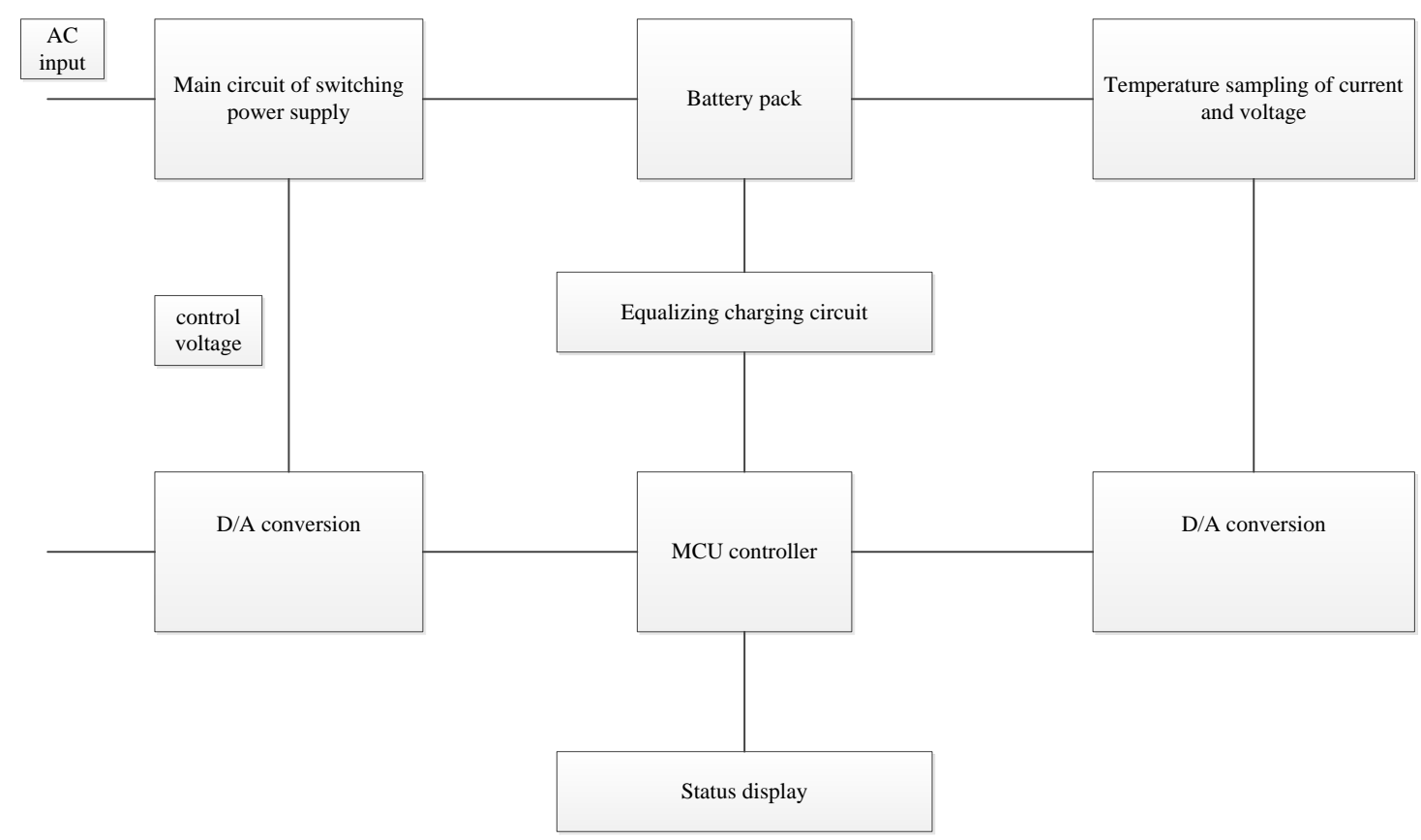

Figure 4: Hardware structure of intelligent charger

The overall design idea is that, after the solar panel input $24 \mathrm{~V}$ direct current, BUCK circuit is used to reduce the voltage. Meanwhile, MCU is powered on to detect the battery charging voltage and judge the charging stage.

The current output voltage and current value are collected. On the one hand, it is displayed in real time after $\mathrm{AD}$ conversion to the single chip microcomputer; on the other hand, it is feedback to the UC3843 regulating circuit.

Then, according to the detected voltage, MCU resets the current and voltage signals to output to the UC3843 regulating circuit as the reference value.
UC3843 compares the feedback value with the reference value, and the resulting deviation realizes the PWM output with different duty ratios after PID operation, and finally realizes the charging control.

The working principle of the step-down circuit uses duty ratio $\mathrm{D}$ to describe the relationship between the on time of switch $\mathrm{S}$ and the working cycle. Duty ratio D can be expressed as Eq.4:

$$
\mathrm{D}=\frac{\mathrm{T}_{\text {on }}}{\mathrm{T}_{\mathrm{S}}}
$$

The differential equation when the switch is closed is as follows: 


$$
\mathrm{V}_{\mathrm{I}}=\mathrm{L} \frac{\mathrm{di}}{\mathrm{dt}}+\mathrm{V}_{0}
$$

For the initial condition of inductive current, the expression of inductive current can be obtained:

$$
\mathrm{i}_{\mathrm{L}}(\mathrm{t})=\frac{\mathrm{V}_{\mathrm{i}}-\mathrm{V}_{0}}{\mathrm{~L}} \mathrm{t}+\mathrm{i}_{\mathrm{L} 0}
$$

When $t=$ Ton, the maximum increment of inductance current is obtained, shown in Eq.7:

$$
\Delta \mathrm{i}_{\mathrm{Lp}}=\frac{\mathrm{V}_{\mathrm{i}}-\mathrm{V}_{0}}{\mathrm{~L}} \mathrm{~T}_{\text {on }}=\Delta \mathrm{i}_{\mathrm{L}}
$$

The differential equation when the switch is off is as follows:

$$
\mathrm{L} \frac{\mathrm{di}_{\mathrm{L}}}{\mathrm{dt}}+\mathrm{V}_{0}=0
$$

The negative increment of inductance current during switch off can be solved.

$$
\Delta \mathrm{i}_{\mathrm{Lm}}=-\frac{\mathrm{V}_{0}}{\mathrm{~L}} \mathrm{~T}_{\text {off }}=-\Delta \mathrm{I}_{\mathrm{L}}
$$

The continuity of inductive current suggests that the net wave momentum of inductive current in a period of steady-state operation should be zero.

$$
\frac{\mathrm{V}_{\mathrm{i}}-\mathrm{V}_{0}}{\mathrm{~L}} \mathrm{~T}_{\text {on }}=\frac{\mathrm{V}_{0}}{\mathrm{~L}} \mathrm{~T}_{\text {off }}
$$

In addition, according to the definition of critical inductance, the critical inductance value of BUCK converter can meet the following requirements:

$$
\frac{\Delta \mathrm{I}_{\mathrm{L}}}{2}=\frac{\mathrm{V}_{0}}{2 \mathrm{~L}} \mathrm{~T}_{\text {off }}=\frac{\mathrm{V}_{0}}{\mathrm{R}}=\mathrm{l}_{0}
$$

By using Eq.11, the calculation equation of the input inductance with the specified inductance current ripple degree can be obtained:

$$
\mathrm{L}=\frac{\mathrm{R}(1-\mathrm{D})}{2 \mathrm{f}_{\mathrm{s}} \delta}
$$

The charging and discharging current of filter capacitor $C$ is the fluctuation value of inductance current, and its fluctuation component will cause the fluctuation of capacitance voltage, and finally form the output ripple. The capacitance voltage fluctuation is calculated as follows:

$$
\begin{aligned}
& \Delta \mathrm{V}_{0}=\frac{1}{\mathrm{C}} \int_{1}^{2} \mathrm{i}_{\mathrm{c}} \mathrm{dt}=\frac{1}{\mathrm{C}}\left(\frac{1}{2} \times \frac{\Delta \mathrm{L}}{2} \times \frac{\mathrm{T}_{\mathrm{S}}}{2}\right)=\frac{\Delta \mathrm{I}_{\mathrm{L}}}{8 \mathrm{C}} \mathrm{T}_{\mathrm{S}} \\
& \Delta \mathrm{V}_{0}=\frac{\mathrm{V}_{0}(1-\mathrm{D})}{8 \mathrm{LCf}_{\mathrm{s}}^{2}}
\end{aligned}
$$

The relationship between circuit parameters and ripple coefficient is obtained by combining the above equations.

$$
\gamma=\frac{\Delta \mathrm{V}_{0}}{\mathrm{~V}_{0}}=\frac{\delta}{4 \mathrm{RCf}_{\mathrm{s}}}
$$

By analyzing the relationship between the input and output of the filter structure, Eq.16 can be obtained:

$$
\frac{u_{0}}{u_{i}}=\frac{\frac{1}{j w c_{2}}}{R+j w l+\frac{1}{j w c_{2}}}
$$

Through analysis and calculation, it is found that there is a restriction relationship between the filter capacitance and the inductance.

$$
\frac{1}{\left|1+\mathrm{jwRC}_{2}-\mathrm{w}^{2} \mathrm{LC}_{2}\right|}=0
$$

The open-loop transfer function can be obtained according to the equivalent diagram of the proportional integral differential circuit.

$$
\mathrm{G}_{1}(\mathrm{~S})=\frac{\left(\mathrm{R}_{1} \mathrm{C}_{1} \mathrm{~S}+1\right)\left(\mathrm{R}_{2} \mathrm{C}_{2} \mathrm{~S}+1\right)}{\mathrm{R}_{1} \mathrm{C}_{2} \mathrm{~S}}
$$

The transfer function of a typical PID regulator is shown in Eq.19.

$$
\begin{aligned}
& G(S)=\frac{1}{P}\left(1+\frac{1}{T_{1} S}+T_{d} S\right) \\
& G(S)=\frac{1}{P}\left(\frac{T_{i} T_{d} S^{2}+T_{i} S+1}{T_{i} S}\right)
\end{aligned}
$$

The open-loop transfer function can be written as Eq.21.

$$
G_{1}(S)=\frac{R_{1} C_{2} R_{2} C_{1} S^{2}+\left(R_{12} C_{1}+C_{2} R_{2}\right) S+1}{R_{1} C_{2} S}
$$

\subsection{Software design}

The control software tool with MCU as the core has the characteristics of flexibility, universality, and reliability. Now the software design idea of the controller is introduced as follows.

Figure 5 shows the software structure frame of the intelligent charger.

First, the main program should complete the initialization of the system to ensure the accuracy of the initial value of the system. A / D samples the voltage and current, and judges the charging stage.

At the beginning of work, MCU sends the default PWM signal, and sets the constant current value to adjust the circuit according to the requirements.

In this stage, MCU will display the corresponding display signal to the digital. When the voltage reaches the limit, MCU will output different PWM waves, which will generate the control voltage $\mathrm{i}$ and enter the constant voltage charging stage.

After entering the constant voltage charging stage, the voltage is constant and the current is continuously reduced.

When the current is reduced to the set value, it enters the floating charging stage.

The controller is designed completely according to the method of structured programming, and the whole program is divided into several subprograms for debugging and checking.

Using C language makes the whole software development simple, fast, and easy to debug. 


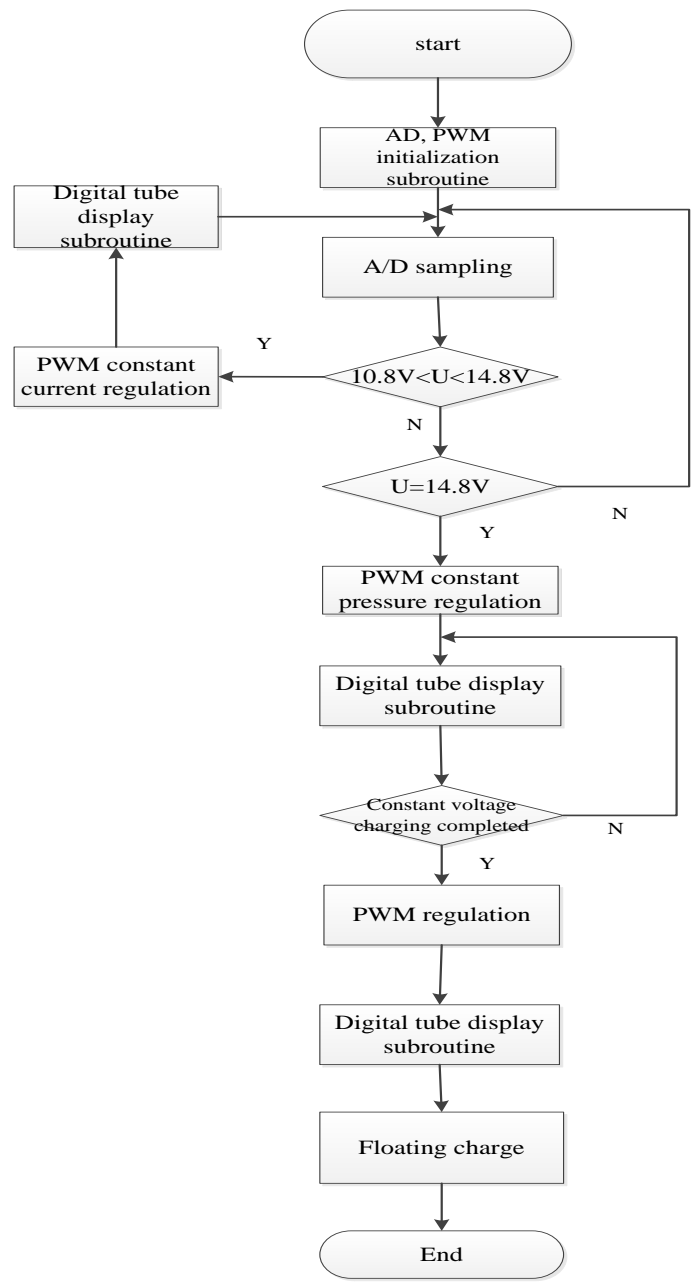

Figure 5: Software structure of intelligent charger

\subsection{Anti-interference design}

When the charging environment of the battery is complex and there are many interference sources, anti-interference measures should be designed to avoid confusion in the charging system when the interference is strong.

Anti-random flight measures. They are mainly designed for the operation of control program. If the charging system is disturbed in the process of operation, it will be easy to make mistakes in the process of program instruction, which will cause the disorder of the program and affect the normal charging process. In order to prevent the program from flying in disorder, single byte instructions should be used as much as possible in the design of the program.

Software filtering measures. Software filtering measures are mainly designed for the tested signal of sampling detection circuit. When the detection signal of the sampling circuit is disturbed, methods such as median method and average method can be used for filtering.

It must be noted that at any stage, the controller must continuously detect the following three key technical indicators: whether the circuit is open, whether the battery is unbalanced, and whether the battery reaches the specified safety voltage.

The open circuit of the battery is mainly judged by detecting the current. And in order to avoid misjudgment, it should be tested repeatedly. When there is an open circuit, it should return to the pretreatment stage. The judgment of open circuit should be carried out when the voltage has reached the predetermined value; otherwise, when the voltage has not reached the predetermined value, the current is relatively small, which may lead to misjudgment.

Balanced charging is another important feature of intelligent electrical appliances. Figure 6 shows the schematic diagram of charging algorithm.

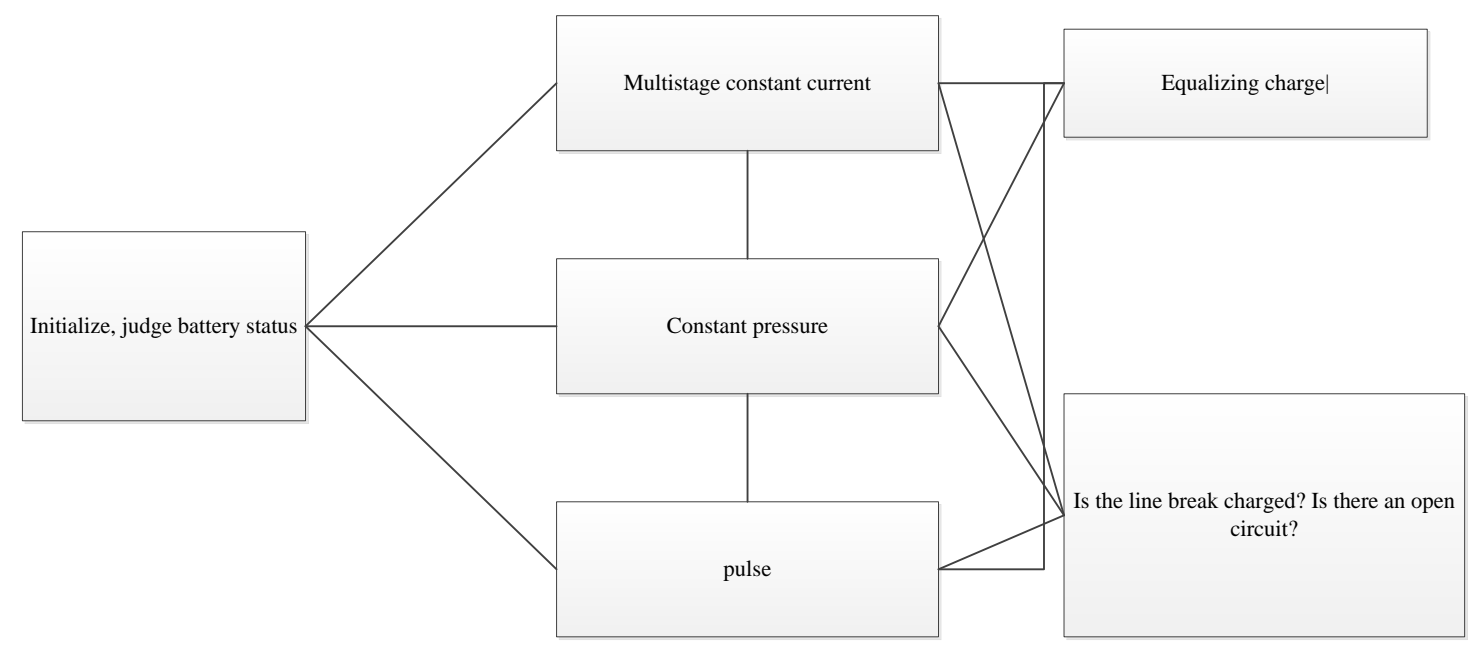

Figure 6: Charging algorithm principle

In the process of charging, due to the different quality of the battery, the voltage of the battery with small capacity and poor quality will increase more after charging the same amount of electricity. If no measures are taken, their voltage will be greatly different, so that one battery will soon reach the 
specified safety voltage, and the charging process will be forced to stop. At this time, the battery with high voltage should be discharged, i.e. balanced charging. In this way, the damaged cells in the battery can be recovered and the charging process can be carried out smoothly. To prevent the battery from being damaged, stop charging immediately when the battery voltage reaches the specified safety voltage; otherwise, the battery will be damaged.

\section{Results and Discussion}

The research on specific energy of electric vehicle under the action of MCU charger is shown in Figure 7. It shows that the specific energy density of leadacid battery of electric vehicle has changed in different trends over time.

Based on STC12C5204AD MCU charger in this paper, the change of specific energy density of battery is relatively small, which is basically maintained at the beginning level. Whereas, the battery specific energy density based on 8xC752 and C504 MCU chargers decreased significantly.

The parameters of specific energy density are very important for the normal operation and life of batteries. Therefore, the charger based on STC12C5204AD MCU has the most prominent protective effect on batteries.

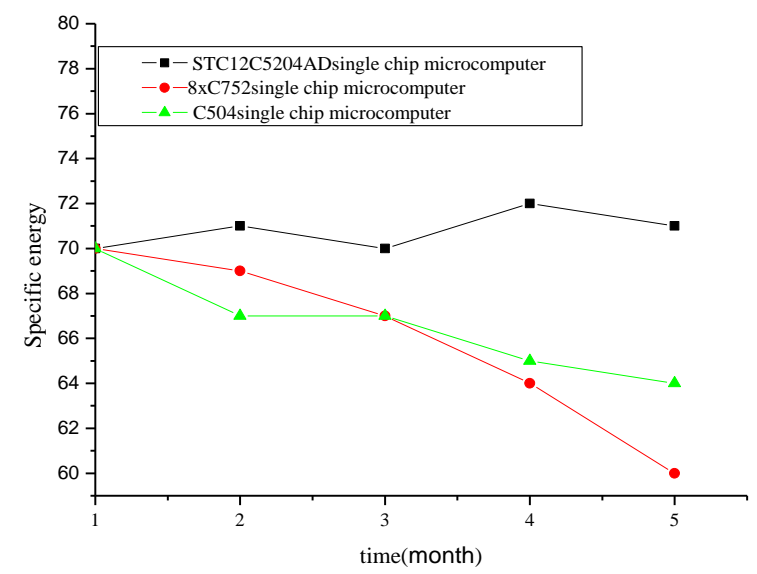

Figure 7: Specific energy of electric vehicle under the action of MCU charger

Figure 8 shows the charging modes of intelligent charger and general charger. The data and trend chart show that the rated voltage required by the battery decreases and declines with the increase of temperature.

In this case, the general charger will not change the charging voltage according to the change of the rated voltage of the battery, and always maintain a certain charging voltage.

This mode of charging will bring great damage to the safety and life of the battery, but the intelligent charger will change its charging voltage according to the change of the rated voltage of the battery, thereby keeping the same step with the rated voltage all the time and protecting the life of the battery to the greatest extent. Hence, the intelligent charger based on STC12C5204AD MCU in this paper is compared with the ordinary charger for the battery protection.

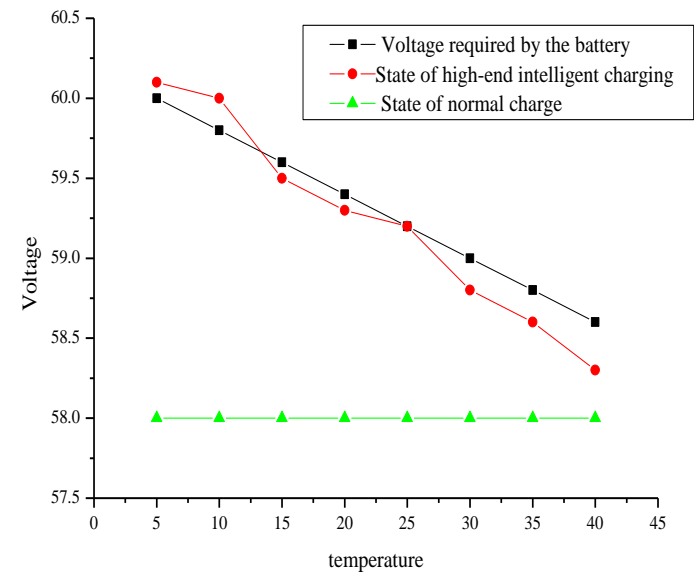

Figure 8: Charging mode of intelligent charger and general charger

Figure 9 shows the research on battery life by the charging times under the action of MCU charger.

It shows that the battery life of chargers based on STC12C5204AD, 8xC752, and C504 MCU decreases with the increase of charging times.

However, the declining trend of battery life based on STC12C5204AD MCU is obviously better than that of other two groups of MCUs. STC12C5204AD singlechip charger has the most prominent protection effect on the battery, obviously delaying the aging process of the battery and prolonging the use time and value of the battery.

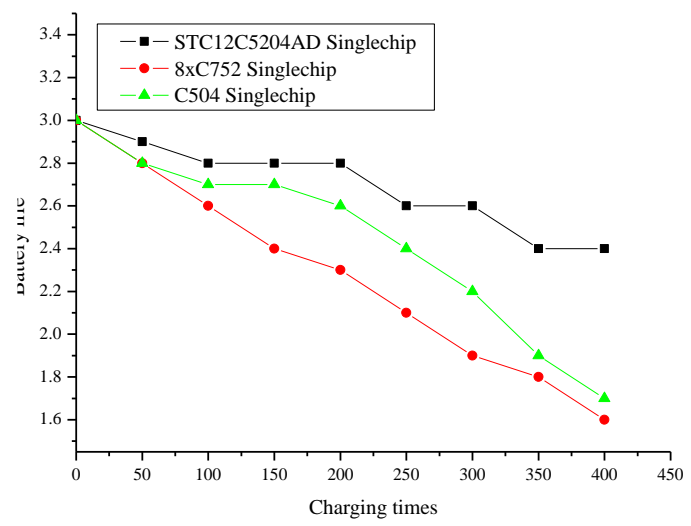

Figure 9: The charging times for battery life under the action of MCU charger

\section{Conclusion}

The specific energy density of battery and the life of battery under the action of STC12C5204AD, 8xC752, and C504 are discussed, and the effect and mechanism of intelligent charger and general charger are explored.

The results show that the battery based on STC12C5204AD MCU charger has little effect on the 
change of specific energy of battery, and the specific energy basically maintains the initial value, with remarkable effect. The STC12C5204AD MCU has the most prominent effect on delaying the decline of battery life compared with the 8xC752 and C504 MCUs under the same charging times. Compared with the general charger, the intelligent charger will change its charging voltage according to the change of battery rated voltage to maintain the straight and maximum protection of battery life. Under the general charging mode, the charging maintains a certain value, without random changes. There are also some deficiencies in the research process.

The conclusion of the research on the charger system of electric vehicles is based on the experimental and theoretical stages, and there will be many factors and problems for the actual field operation, and the results are slightly less convincing. Nevertheless, the research in this paper is qualitative and provides valuable reference for the further research on electric vehicles.

\section{References}

[1] Jiang G., He H., Yan J, et al. (2018) Multiscale convolutional neural networks for fault diagnosis of wind turbine gearbox. IEEE Transactions on Industrial Electronics, 66(4), 3196-3207.

[2] Peeters C., Guillaume P., Helsen J. (2018) Vibration-based bearing fault detection for operations and maintenance cost reduction in wind energy. Renewable Energy, 116, 74-87.

[3] Dao P. B., Staszewski W. J., Barszcz T, et al. (2018) Condition monitoring and fault detection in wind turbines based on cointegration analysis of SCADA data. Renewable Energy, 116, 107-122.

[4] Feng Z., Qin S., Liang M. (2016) Time-frequency analysis based on Vold-Kalman filter and higher order energy separation for fault diagnosis of wind turbine planetary gearbox under nonstationary condition. Renewable Energy, 85, 45-56.

[5] Chen J., Pan J., Li Z, et al. (2016) Generator bearing fault diagnosis for wind turbine via empirical wavelet transform using measured vibration signals. Renewable Energy, 89, 80-92.

[6] Cao M., Qiu Y., Feng Y, et al. (2016) Study of wind turbine fault diagnosis based on unscented Kalman filter and SCADA data. Energies, 9(10), 847.

[7] Maheswari R. U., Umamaheswari R. (2017) Trends in non-stationary signal processing techniques applied to vibration analysis of wind turbine drive train-A contemporary survey. Mechanical Systems and Signal Processing, 85, 296-311.

[8] Astolfi D., Scappaticci L., Terzi L. (2017) Fault diagnosis of wind turbine gearboxes through temperature and vibration data. International Journal of Renewable Energy Research (IJRER), $7(2), 965-976$

[9] Zhao H., Liu H., Hu W, et al. (2018) Anomaly detection and fault analysis of wind turbine components based on deep learning network. Renewable energy, 127, 825-834.

[10] Qiu Y., Feng Y., Sun J, et al. (2016) Applying thermophysics for wind turbine drivetrain fault diagnosis using SCADA data. IET Renewable Power Generation, 10(5), 661-668.

[11] Romero A., Soua S., Gan T. H, et al. (2018) Condition monitoring of a wind turbine drive train based on its power dependant vibrations. Renewable energy, 123, 817-827.

[12] de Azevedo H. D. M., Araújo A. M., Bouchonneau N. (2016) A review of wind turbine bearing condition monitoring: State of the art and challenges. Renewable and Sustainable Energy Reviews, 56, 368-379.

[13] Chen H., Chen P., Chen W, et al. (2017) Wind turbine gearbox fault diagnosis based on improved EEMD and Hilbert square demodulation. Applied Sciences, 7(2), 128.

[14] Saidi L., Ali J. B., Bechhoefer E, et al. (2017) Wind turbine high-speed shaft bearings health prognosis through a spectral Kurtosis-derived indices and SVR. Applied Acoustics, 120, 1-8.

[15] Guo J., Lu S., Zhai C, et al. (2018) Automatic bearing fault diagnosis of permanent magnet synchronous generators in wind turbines subjected to noise interference. Measurement Science and Technology, 29(2). 025002.

[16] Hosseinzadeh M., Rajaei Salmasi F. (2016) Analysis and detection of a wind system failure in a micro-grid. Journal of Renewable and Sustainable Energy, 8(4), 043302.

[17] $\mathrm{Hu}$ Y., Tu X., Li F, et al. (2018) Joint high-order synchrosqueezing transform and multi-taper empirical wavelet transform for fault diagnosis of wind turbine planetary gearbox under nonstationary conditions. Sensors, 18(1), 150. 